\title{
Improving Safety \& Ride Comfort through Dual Suspension System in Road Vehicles
}

\author{
Ch. Kumar ${ }^{1}$, Dr. Z. A. Arain², S. A. RaZA ${ }^{3}$, S. Kumar ${ }^{4}$, S. Z. ul Abedin Abidi ${ }^{5}$, Ch. Lal ${ }^{6}$ \\ 1-6 Faculty of Engineering, Science and Technology, Indus University, Karachi, Sindh, Pakistan \\ chandar.kumar@indus.edu.pk, arainzahid@hotmail.com, Ahmedsyed64@gmail.com \\ sureshkumar@indus.edu.pk,zain.abidi@indus.edu.pk, chaman.lal@indus.edu.pk
}

\begin{abstract}
The essential objective of the proposed suspension framework is to limit the undesired disturbance of a vehicle because of uneven surfaces on streets. This framework in vehicles is comprise of some fundamental components like dampers and springs which ingests the stuns that are instigated by unusual streets. In any case, these frameworks can just work up to a predefined constrain. Vehicle suspension frameworks are planned by taking the solace capacity of a traveler in record and to enhance the street grasp on each surface.
\end{abstract}

This examination proposes an idea for structuring a superior suspension arrangement of vehicles to upgrade the nature of the drive without corrupting the dependability of the vehicle plan. In this exploration, a mix of two suspension framework is presented in which one goes about as an essential suspension while different fills in as an optional framework that can adequately lessen the vibrations. This mix upgrades the customary framework that is right now introduced in vehicles without bargaining on solace and street hold.

Keywords: Primary Suspension, Secondary Suspension, (DoF) Degree of Freedom, Road Vehicles

\section{Introduction}

The motivation behind the suspension framework is to enhance the security, dealing with and the ride nature of the vehicles. Regular vehicle suspension frameworks accomplish this through uninvolved means utilizing springs and dampers [1]. When planning vehicle suspensions, the double target is to limit the vertical powers transmitted to the traveller, and to augment the tire - to-street contact for taking care of and wellbeing [1]. For steadiness and taking care of stiffer suspension is required while, for ride comfort relatively delicate suspension is reasonable [2], or, in other words exchange off between ride quality and the strength of the vehicle. With the end goal to determine this issue semi-dynamic and dynamic suspension have been proposed by numerous specialists [3-5], which require costly components, for example, factor dampers, sensors and actuators, and a control instrument that thusly builds the general assembling cost of the vehicles [6, 7].In street vehicles the suspension frameworks comprise just of single stage, as appeared in Fig. 1, which fills for each of the three needs (i.e. soundness, dealing with and ride comfort).However trains have twofold suspension framework as appeared in Fig. 2.shows the arrangement of the most present day traveller conveying railroad vehicles. Every auto comprises of two intruders, every one of which has two arrangements of wheels. The motivation behind the bogie is to convey the heaviness of a vehicle along the track at the required speed and with a high 
level of wellbeing. In doing as such, and to the extent practicable, it secludes the vehicle from dynamic powers and vibrations coming about because of movement. The auto body is associated with intruders by means of suspensions (auxiliary suspension), the motivation behind which is to give great ride quality by detaching the auto body from vibrations initiated by track abnormalities. The wheel sets are associated with the bogie through essential suspensions, whose components are significantly stiffer than in the optional suspension framework and are intended to fulfil the vehicle's strength and direction necessities [2].

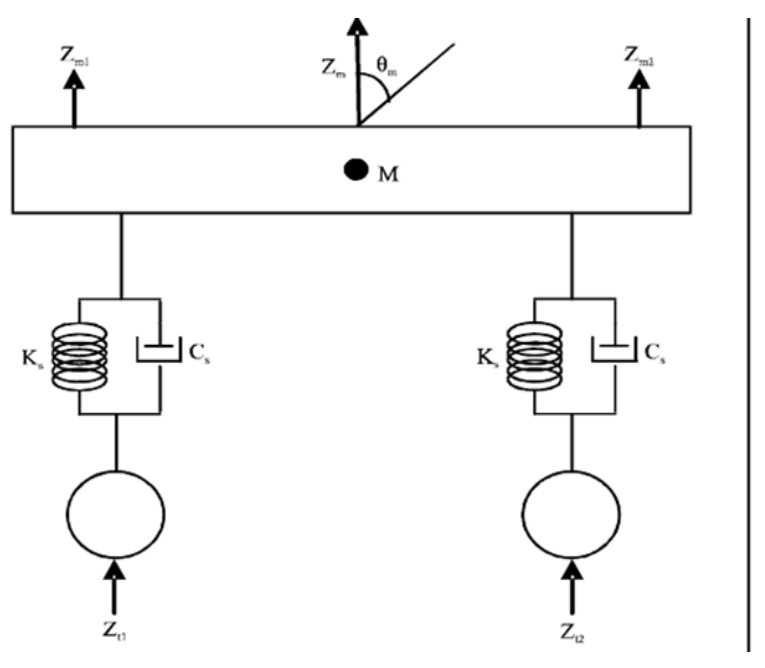

Figure 1. Conventional Suspension System

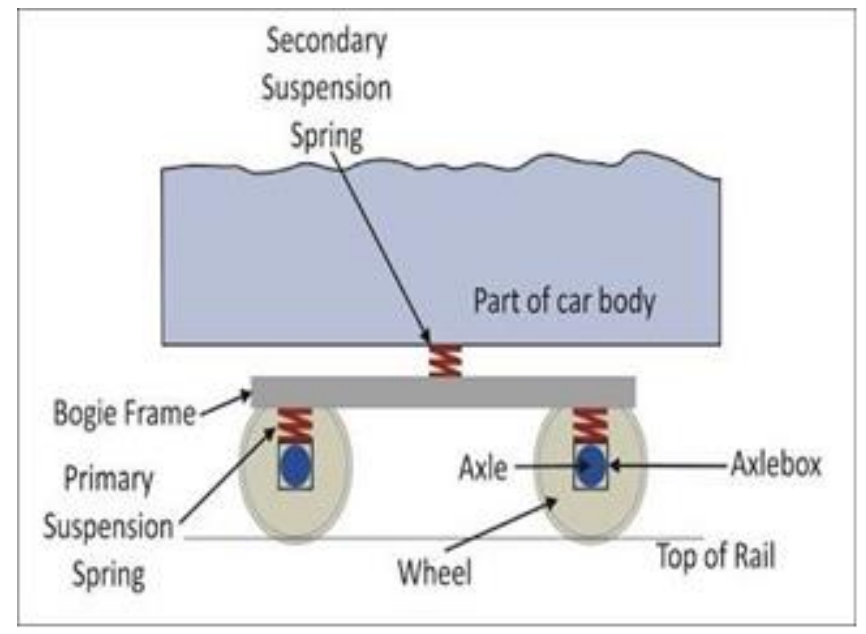

Figure 2. Primary and Secondary Suspension in Railway Vehicles

In this paper a suspension framework like the railroad suspension, as appeared in Fig. 3, is proposed for street vehicles. The one end of essential and optional suspension components are mounted on a middle of the road mass called body outline. The motivation behind the essential suspensions to enhance the steadiness of the vehicle by guaranteeing greatest tire-street contact on sharp bends and the reason for optional suspension is to confine the vehicle body from street abnormalities .The capability of this exploration work is displayed utilizing a straightforward model with 4 DoF (Degrees of Freedom). The work can additionally be reached out for a full vehicle show by presenting more DoF. 


\section{Modelling of System Dynamics}

In this examination just those DoF are viewed as which are pertinent to ride comfort (i.e. Bob movement and Pitch Angle) other DoF have little impact on ride comfort in this way, are disregarded in this investigation. A point by point graph of the street vehicle with essential and optional suspension is appeared in Fig. 4, where Zt1 and Zt2 are the skip movements connected by the way to the front and back wheels individually. $\mathrm{Z}$ is the resultant

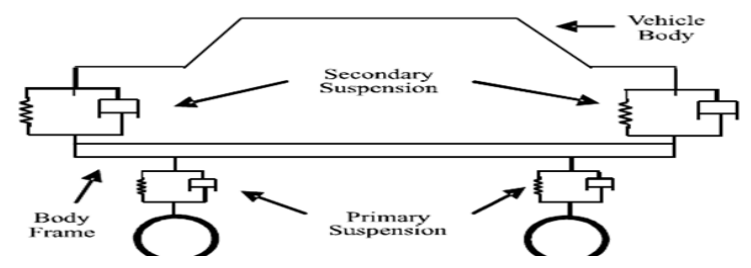

Figure 3. Proposed primary and secondary suspension in road Vehicles

Bob movement of the vehicle body and $\mathrm{K}$ is the contribute edge radians. $\mathrm{L}$ is the separation among front and back wheels, Z3 and Z4 are the skip movements of the body outline. M is the mass of the vehicle body; $\mathrm{m} 1$ and $\mathrm{m} 2$ are the majority of the body outline anticipated to front and back wheels individually. $\mathrm{Z} 1$ and $\mathrm{Z} 2$ are the vertical movements of the vehicle body because of pitch point. The model is spoken to by Equations (1-10) and images are clarified in Appendix-I.

$$
f 1=C 1(\mathrm{z} \cdot 1-\mathrm{z} \cdot 3)+K 1(\mathrm{z} \cdot 1-z 3)
$$

$$
f 2=C 2(\mathrm{z} \cdot 2-\mathrm{z} \cdot 4)+K 2(z 2-z 4)
$$

$$
\mathrm{m} 3 \mathrm{z} \cdot 3=C 1(\mathrm{z} \cdot 1-\mathrm{z} \cdot 3)+K 1(z 1-z 3)
$$

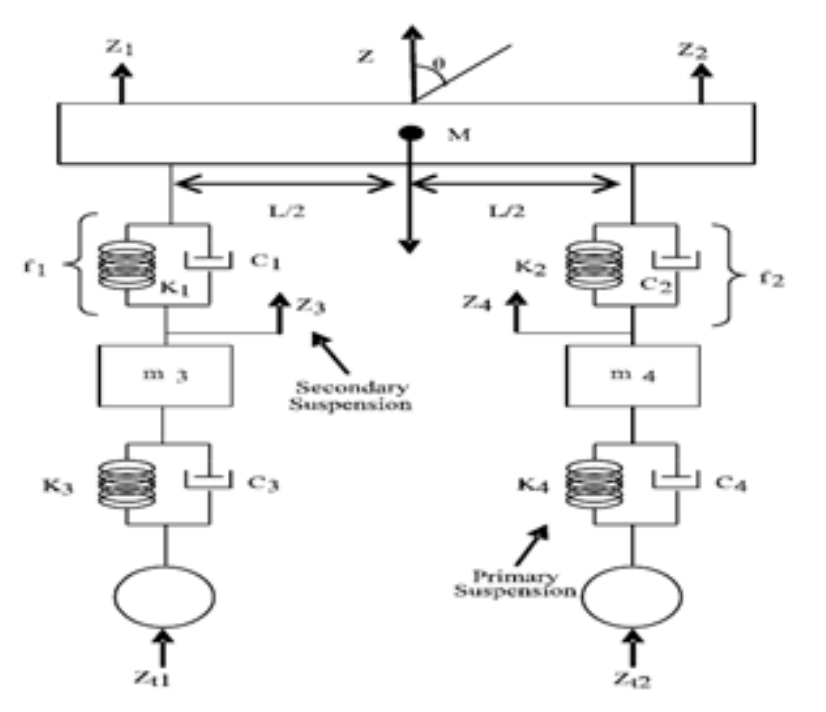

Figure 4. Proposed Primary and Secondary

Suspension (4 Dof) 


$$
m 4=z " 3=C 1(z \cdot 1-z \& 3)+K 1(z 1-z 3)
$$

It is expected that the vehicle is going on straight road therefore the pitch edge would be a little esteem. Utilizing the little edge estimation rest of the conditions can be composed as pursues.

$$
\begin{gathered}
z=z+\mathrm{L} / 2 * \\
\mathrm{z}=\mathrm{z}-\mathrm{L} / 2 * \\
\mathrm{~T} 1=\mathrm{L} / 2
\end{gathered}
$$

It is normal that the skip movement $\mathrm{Z}$ of the vehicle body would be significantly littler than the vertical aggravations ( $\mathrm{Zt} 1$ and $\mathrm{Zt} 2$ ) connected by the street. It is additionally anticipated that that accordingly would any sudden unsettling influence, for example, a pothole in the street, the body movement would be smooth as contrast with regular suspension framework.

\section{Result and Discussion}

\subsection{Comparison between Conventional and Proposed Suspension System (SS):}

By looking at the execution of the Conventional and Purposed suspension framework utilizing essential and optional suspension strategy it is unmistakably demonstrates that the purposed suspension can give bring down adequacy and quicker setting time. Suspension Travel for the two sorts of Road Profiles (Step and White Noise) can diminish the sufficiency and settling time contrast with customary suspension framework. Body Displacement likewise enhances with traditional suspension framework and the setting time is quick. Body Displacement is utilized to speak to ride quality. The reaction of proposed suspension model to the street's unpleasantness is again much superior to the ordinary one. Examination among Conventional and proposed suspension framework against both advance and harshness.

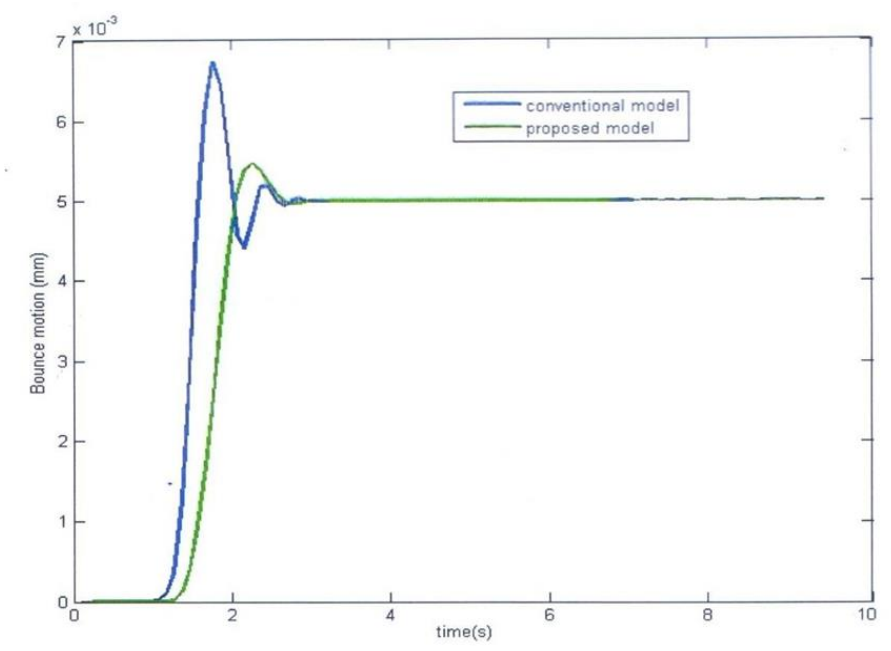

Figure 5. Step response of proposed and conventional model (Bounce) 
Demonstrates that the skip movement of the traditional framework and proposed show vehicle body against the progression input. The bob movement of the vehicle body overshoots and undershoots few times previously it is settled down

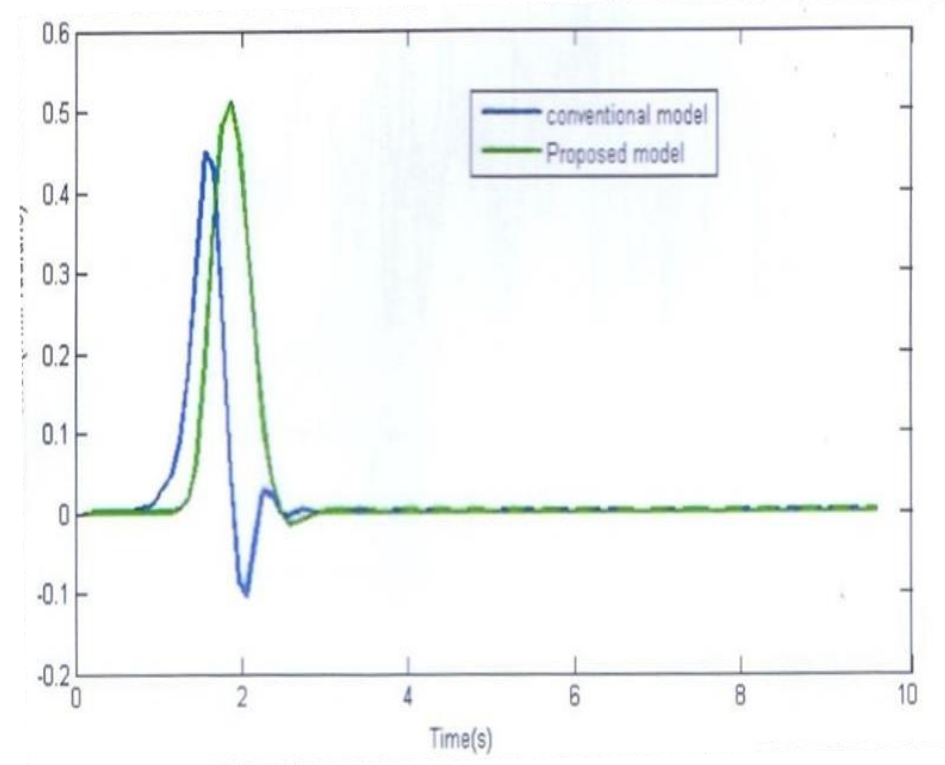

Figure 6. Step response of proposed and conventional model (Pitch)

see that the bounce motion of proposed suspension system doesn't increase $+\mathrm{mm}$, whereas, the bounce motion of the system now and then increase $20 \mathrm{~mm}$, as shown above

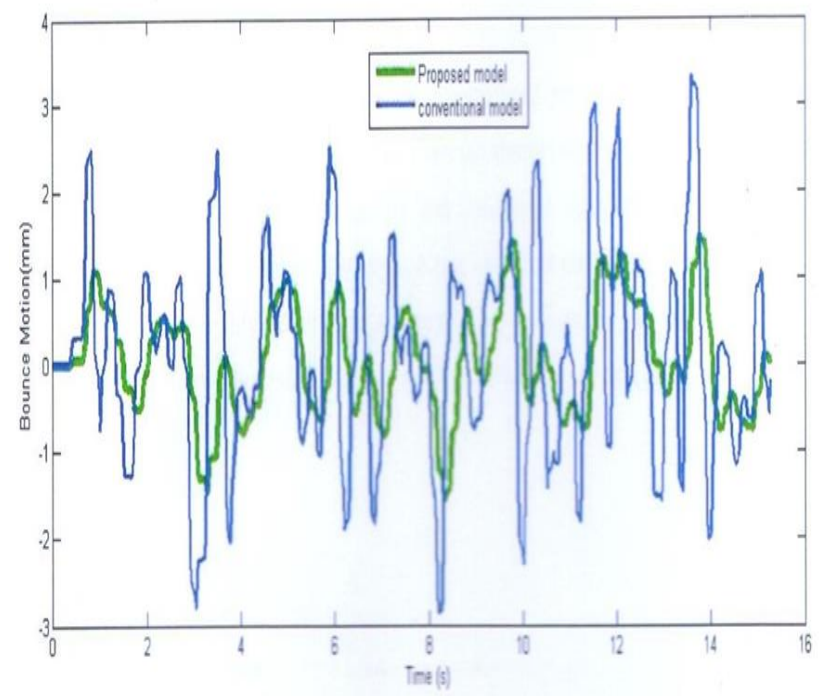

Figure 7. Bounce comparison of proposed and conventional model 


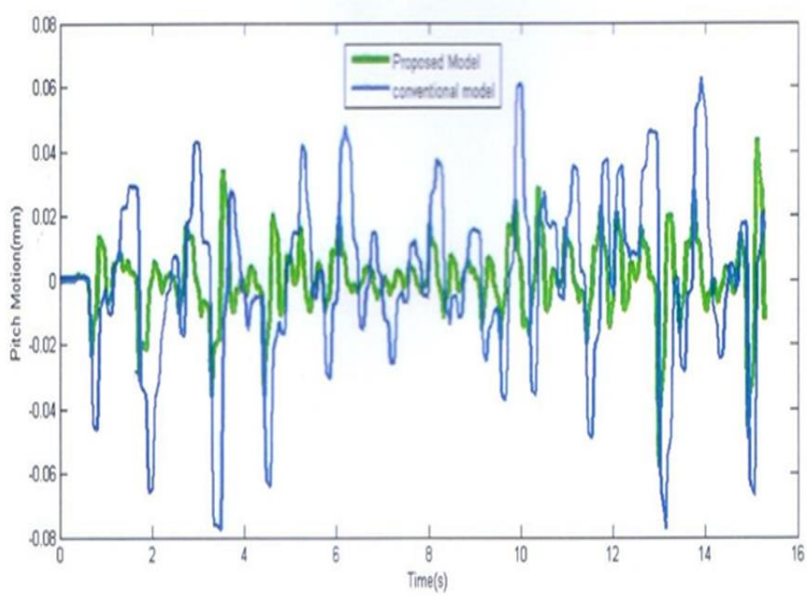

Figure 8. Pitch comparison of proposed and conventional model

\section{Conclusion}

The possibility of this examination depends on the usage of double suspension framework which upgrades the treatment of the vehicle and ride quality and solidness on harmed surfaces of the streets. Generally the suspension arrangement of vehicles achieves this control by a solitary stage aloof or semidynamic and dynamic framework utilizing blends of dampers and springs. As it is as of now talked about that for better security a stiffer suspension framework is required, though, for better solace and hold, a delicate suspension framework is required. So a superior exchange off was expected to adjust between both solace and solidness and introducing double suspension framework was displayed alongside the scientific demonstrating and re-enactment results

\section{References}

[1] Imtiaz Hussain, Jawaid Daudpoto \& Ali Asghar Memon, "Introducing Dual Suspension System in Road Vehicles", Mehran University Research Journal of Engineering \& Technology, Volume 32, No. 1, January, 2013..

[2] Rosheila Binti Darus, "Modelling and Control of Active suspension for a Full CarModel”, Faculty of Electrical Engineering University of Technology Malaysia, May, 2008.

[3] Vijay R. Patil, Girish B. Pawar\& Suresh A. Patil "A Comparative Study between the Vehicles" Passive and Active Suspension - A Review, International Journal of Engineering Research and Applications (IJERA) Vol. 3, Issue 1, January - February, 2013.

[4] Claudiu Velentin Suciu, Tsubasa Tobiishi \& Ryouta Mouri "Modelling and Simulation of a Vehicle Suspension with Variable Damping and Elastic Properties versus the Excitation Frequency", International Conference on P2P, Parallel, Grid, Cloud and Internet Computing, 2011.

[5] Jianmin Sun \&Qingmei Yang "Compare and Analysis of Passive and Active Suspension under Random Road Excitation", Proceedings of the IEEE International Conference on Automation and Logistics Shenyang, China August 2009

[6] Yaojung Shio, Chun-Chi Lai \& Quang-Anh Nguyen "The Analysis of Semi-Active Suspension System", SICE Annual Conference 2010 August 18-21, ,the Grand Hotel, Taipei, Taiwan2010,.. 
[7] Yi-Pin Kuo, Neng-Sheng Pai, Jui-Sheng Lin \&Ching-Yang Yang "Passive Vehicle Suspension System Design Using Evolutionary Algorithm” 2008.

[8] Saiful Anuar Abu Bakar, Ryosuke Masuda, Hiromu Hashimoto, Takeshi Inaba, Hishamuddin Jamaluddin, Roslan ABD, Rahman \& Pakharuddun Mohd, Samin "Ride Comfort Performance of Electric Vehicle Conversion with Active Suspension System", SICE Annual Conference 2012 August 20-23, , Akita University, Akita, Japan2012.

[9] Qingmei Yang \& Jiammin Sun "A Novel Fuccy Controller to Improve Comfort Feature of Vehicle", 2009 IEEE.

[10] U Can, Wang Weirui, Shi Haoran\& Zhang Weiqian "Research on the stability of vehicle suspension excited by the road surface profile", Second International Conference on Computer Modeling and Simulation,2010.

[11] Zheng Yinhuan "Research on fuzzy logiccontrol of vehicle suspension system", IEEE2010..

[12] Dowds, P., \& O'Dwyer, A., “Modelling and Control of a Suspension System for Vehicle Applications", Proceedings of the 4th Wismarer Automatisierungs Symposium, Wismar, Germany, September 22-23.

[13] Donald, L., \& Margolls, "The Response of Active and Semi-Active Suspensions to Realistic Feedback Signals", International Journal of Vehicle Mechanics and Mobility, Volume 11, Nos, 56, pp, 267-282, July, 2007.

[14] Martins, l., Esteves, J., Marques, GD., \& Pina Da Silva, F., "Permanent Magnet and Linear Actuators Applicability in Automobile Active Suspensions", IEEE Transactions on Vehicular Technology, Volume 55, No. 1, pp. 86-94, January, 2006.

[15] Chen, Yi, “Vehicle Suspension System Modelling”. 13th Sep, 2006.

[16] Kim C., Ro P.I, “An Accurate Full car Ride Model Using Model Reducing Techniques”, Journal of Mechanical Design. 2002. 124:697-705.. 